\title{
1 Meta analysis of variant predictions in congenital adrenal 2 hyperplasia caused by mutations in CYP21A2
}

Mayara J. Prado ${ }^{1,2,3,4}$, Rodrigo Ligabue-Braun ${ }^{5}$, Arnaldo Zaha ${ }^{1,2} \ddagger$, Maria Lucia Rosa Rossetti ${ }^{1,4} \ddagger$, Amit

$$
\text { V. Pandey }{ }^{3,4} ¥
$$

$\ddagger$ These authors contributed equally to this work.

${ }^{1}$ Graduate Program in Cell and Molecular Biology, Universidade Federal do Rio Grande do Sul (UFRGS), Porto Alegre CEP 91501-970, Brazil. ${ }^{2}$ Center for Biotechnology, Universidade Federal do Rio Grande do Sul (UFRGS), Porto Alegre CEP 91501-970, RS, Brazil. ${ }^{3}$ Department of Biomedical Research, University of Bern, Bern 3010, Switzerland. ${ }^{4}$ Pediatric Endocrinology Unit, Department of Pediatrics, University Children's Hospital Bern, Bern 3010, Switzerland. ${ }^{5}$ Department of Pharmacosciences, Universidade Federal de Ciências da Saúde de Porto Alegre (UFCSPA), Porto Alegre CEP 90050-170, Brazil. ' Graduate Program in Molecular Biology Applied to Health, Universidade Luterana do Brasil (ULBRA), Canoas CEP 92425-020, Brazil.

ORCiD numbers: M.J.P (0000-0003-0647-4429), R.L-B. (0000-0002-2555-9754), A.Z. (0000-0001-6336474X), M.L.R.R (0000-0002-9672-9394), and A.V.P (0000-0001-8331-5902).

Short title: Meta-analysis of variant predictions in CYP21A2 deficiency. 


\section{Corresponding author's contact information:}

Name: Mayara Jorgens Prado

Address: Federal University of Rio Grande do Sul (UFRGS), Biotechnoloy center 9500, Bento Gonçalves

E-mail: mayjorgens@hotmail.com.

Name and address of person to whom reprints should be addressed: Same as corresponding author.

Funding: This research was funded by a Swiss Government Excellence Scholarship (ESKAS), grant number 2020.0209, and a Conselho Nacional de Desenvolvimento Científico e Tecnológico Scholarship (CNPq).

Disclosure: The authors declare no conflict of interest. The funders had no role in the design of the study; in the collection, analyses, or interpretation of data; in the writing of the manuscript, or in the decision to publish the results. 
Abstract:

Context: CYP21A2 deficiency represents 95\% of congenital adrenal hyperplasia cases (CAH), a group of genetic disorders that affect steroid biosynthesis. The genetic and functional analysis provides critical tools to elucidate complex $\mathrm{CAH}$ cases. One of the most accessible tools to infer the pathogenicity of new variants is in silico prediction.

Objective: Analyze the performance of in silico prediction tools to categorize missense single nucleotide variants (SNVs) of the CYP21A2.

Methods: SNVs of the CYP21A2 characterized in vitro by functional assays were selected to assess the performance of online single and meta predictors. SNVs were tested separately or in combination with the related phenotype (severe or mild CAH form). In total, 103 SNVs of the CYP21A2 (90 pathogenic and 13 neutral) were used to test the performance of 13 single-predictors and four meta-predictors.

Results: SNVs associated with the severe phenotypes were well categorized by all tools, with an accuracy between 0.69 (PredictSNP2) and 0.97 (CADD), and Matthews' correlation coefficient (MCC) between 0.49 (PoredicSNP2) and 0.90 (CADD). However, SNVs related to the mild phenotype had more variation, with the accuracy between 0.47 (S3Ds\&GO and MAPP) and 0.88 (CADD), and MCC between 0.18 (MAPP) and 0.71 (CADD).

Conclusion: From our analysis, we identified four predictors of CYP21A2 pathogenicity with good SNVs' in CYP21A2.

Keywords: online prediction; CYP21A2; mutation analysis; pathogenicity prediction. 


\section{Introduction}

55

56

57

One of the most common autosomal recessive genetic disorders is the impairment of the steroid 21hydroxylase (CYP21A2). This CYP21A2 deficiency represents about $95 \%$ of cases in the congenital adrenal hyperplasia (CAH), a group of enzymatic disorders that affect cortisol biosynthesis. The CYP21A2 enzyme is a member of the cytochrome P450 superfamily (CYPs) and catalyzes the conversion of $17 \alpha$-hydroxyprogesterone (17OHP) into 11-deoxycortisol and progesterone into 11 deoxycorticosterone. Other enzymes subsequently convert these steroids into cortisol and aldosterone, respectively ${ }^{1}$.

Clinically, the CYP21A2 deficiency in humans has a wide spectrum of phenotypes, from severe to mild or asymptomatic ${ }^{2,3}$. The classical severe CAH has salt-wasting (SW) and simple-virilizing (SV) forms. The classical SW form has no enzyme activity and is related to severe virilization and electrolyte dysregulation. In contrast, the classical SV form has enough residual enzyme activity to prevent adrenal crisis ${ }^{2}$. The mild CAH is the non-classical (NC) form of CAH and has a partial CYP21A2 activity associated with hyperandrogenism and mild late-onset $\mathrm{CAH}^{3}$. Furthermore, there is a relatively good genotype-phenotype correlation for CYP21A2 deficiency, which allows the categorization of variants according to the residual enzyme activity (obtained from in vitro studies) and their expected phenotype ${ }^{3}$. The classical CAH has less than $10 \%$ of wild-type (WT) enzyme activity in $95 \%$ of the cases, while the NC form has an activity between $10-78 \%$ of the WT in $90 \%$ of the cases. ${ }^{4}$.

The CYP21A2 gene is a tandemly arranged module (RCCX: RP-C4-CYP21-TNX) and shows $96-98 \%$ of sequence identity with its pseudogene, CYP21A1P ${ }^{5}$. These features make the CYP21A2 gene analysis a complex endeavor, with many different types of mutations - from single nucleotide variants (SNVs) to genetic rearrangements - and further complicated by the fact that most carriers have compound heterozygous mutations ${ }^{3}$. However, only ten mutations described in the general population are sampled by CYP21A2 deficiency screening programs. The whole gene sequence analysis by Sanger 
sequencing is an alternative method for exceptional cases due to the cost and time-consuming nature of such studies ${ }^{6,7}$.

So far, with the whole CYP21A2 gene sequencing, genetic studies have reported more than 1,300 variants for the CYP21A2 gene. Out of the 230 variants reported as affecting human health, 153 are missense variants ${ }^{4}$. The advancement of next-generation sequencing (NGS) to analyze a large number of genes has facilitated the detection of rare single nucleotide variants (SNVS) and single nucleotide polymorphisms (SNPs). A few years ago, this technology was not applied to screen the CYP21A2 gene defects due to its high sequence identity with CYP21A2P which hampers the proper analysis of this genomic region ${ }^{5}$. However, recently some groups have found alternative ways to perform NGS for the CYP21A2 gene through a combination with other methods, such as Multiplex ligation-dependent probe amplification ${ }^{8,9}$. These genetic analysis strategies of the CYP21A2 gene with the NGS technology represent a promising tool for the future, opening the window to identify new variants while improving the diagnosis of CYP21A2 deficiency, and establishing a more reliable estimate of mutation frequencies.

The gold standard for the characterization of new CYP21A2 variants is in vitro functional assay. However, this approach takes too much time, and it is not viable for all-new variants detected. One of the most accessible tools to predict the pathogenicity of variants is the in-silico analysis, which usually has free access, a friendly interface, and provides quick results. Many online predictors are available that have different features and approaches, from single characteristic analysis to meta-predictors with different compositions and algorithms. Some studies have shown the general performances of these tools against a whole database with few predictors ${ }^{10,11}$. However, studies with variants on protein-specific analysis showed that general analysis results cannot be extrapolated for all proteins as each protein has unique characteristics, which is a key limitation of predictor programs ${ }^{12-14}$. Therefore, it is essential to be careful when choosing the prediction tools and to consider their variable accuracies for each gene ${ }^{11}$. 
Here we have done a meta-analysis for the performance of online predictor tools to classify missense SNVs of the CYP21A2. Missense SNV is the most common group of variants in the human genome, one at every kilobase. In the CYP21A2 gene, this type of SNV represents about $60 \%$ of the CYP21A2 variants in The Human Gene Mutation Database (HGMD, RRID:SCR_001888) ${ }^{6}$ and 65\% of those affecting human health ${ }^{4}$. Additionally, missense SNV is one of the hardest variant types for interpretation ${ }^{4,15,16}$. In total, we analyzed 17 predictors with multiple algorithms, approaches and datasets. Thirteen of these were based on single features: CADD (RRID:SCR_018393) ${ }^{17}$, ConSurf (RRID:SCR_002320) ${ }^{18}$, DANN ${ }^{19}$, FATHMM ${ }^{20}$, MAPP (RRID:SCR_014375) ${ }^{21}$, MutPred2 (RRID:SCR_010778) ${ }^{22}$, PANTHER-PSEP (RRID: SCR_005145) ${ }^{23}$, PhD-SNP' (RRID: SCR_010782) ${ }^{24}$, PolyPhen-2 (RRID:SCR_013189) ${ }^{25}$, PROVEN (RRID: SCR_002182) ${ }^{26}$, SIFT (RRID:SCR_012813) ${ }^{27}$, SNAP2 (RRID:SCR_002127) ${ }^{28}$, and SNPs\&GO (RRID:SCR_005788) ${ }^{29}$. Four meta-predictors: PredictSNP ${ }^{30}$, PredictSNP2 ${ }^{31}$, Meta-SNP ${ }^{32}$, and S3Ds\&GO ${ }^{33}$ ) (Figure 1). We excluded nonsense and frameshift variants from our analysis since they have specific settings in many predictors and a high agreement ratio between tools.

\section{Materials and Methods}

\section{SNVs selection and categorization}

To select CYP21A2 missense single nucleotide variants (SNVs) with clinical significance, we used the list of variants reported to affect human health, as reviewed by Simonetti et al ${ }^{4}$. Complementarily, we searched for SNVs reported by dbSNP, Ensembl, and GeneCards applying the following filter when present: "missense", clinical significance "pathogenetic" or "benign", "without conflicting interpretation", and "human or homo sapiens". We excluded nonsense and frameshift mutations. In addition, we performed a cross-check of the databases with original articles or reviews to remove variants without the enzyme activity data available. 
To standardize the effect of the SNVs selected, we categorized them into three groups according to the CYP21A2 activity for at least one of the steroid substrates: classical (CL), nonclassical (NC), and neutral. The CL group has SNVs with the CYP21A2 activity level $<10 \%$ relative to $\mathrm{WT}$, the NC group has SNVs with the activity level between $>10 \%$ and $<78 \%$ relative to WT, and the neutral group has SNV with the enzyme activity $>78 \%$ relative to WT. The CAH group is composed of all SNVs from the CL and NC. The mean and standard deviation (SD) of enzyme activity were calculated for each steroid and mutation group.

To choose predictors with different features, we reviewed the literature for software applied to in silico analysis of SNP or SNVs. Predictors used in more than two studies by different research groups or significant performance in a large study were selected. In addition, we filtered for tools with free access and online availability, thus not requiring local powerful computational resources. Characteristics of each predictor chosen are shown in Table S1-S2.

The default setting for missense mutation was used on all predictors. However, when there was no set instruction for that purpose highlighted for the program, we followed the developers' recommendation from the tutorial or original paper. In addition, three scores were extracted indirectly from meta-predictors: MAPP (v.28.6.2005) and SIFT (v.4.0.4) scores were obtained from

147 PredictSNP, and DANN (v.1.2) score from PredictSNP2. For statistical purposes, we standardized two variables for the outputs of all the predictors: "Damage" for SNVs with the potential to affect CYP21A2 and "neutral" for SNVs with no or very low potential to affect the enzyme. The following outputs were 
(weighted) and PhD-SNP;; "deleterious" message to PredictSNP, PredictSNP2, MAPP, and SIFT; score

Otherwise, we classified the outputs as "neutral".

We analyzed the performance of each predictor in two ways. First, to assess the performance to discriminate the effects of CYP21A2 SNVs, we compared SNVs of the CAH group with the neutral

\section{Statistical methods}

We considered true positive (TP) result for correct "damage" prediction, true negative (TN) for correct

$171 N P V=\frac{T N}{T N+F N}$ 
Finally, we applied the MCC to measure the two-class quality (harmful and neutral). This method is suitable for imbalanced data and has been used to evaluate in silico prediction approaches. MCC score ranges from 1 (perfect prediction) to -1 (total disagreement between the results predicted and observed), with 0 being no better than random prediction (Eqn. 6) (Chicco et al., 2021).

$\mathrm{MCC}=\frac{T P X T N-F P X F N}{\sqrt{(\mathrm{TP}+\mathrm{FP})(\mathrm{TP}+\mathrm{FN})(\mathrm{TN}+\mathrm{FP})(\mathrm{TN}+\mathrm{FN})}}$

Results

From variants in the CYP21A2 gene reported in the literature and databases, we selected missense SNVs with clinical significance (criteria described in section 4.1). We obtained 96 valid SNVs out of 299 missense variants in the list [4], 85 out of 614 missense variants in dbSNP, 66 out of 459 missense variants in Ensembl, 45 out of 71 missense variants in GeneCards, 47 out of 83 missense variants in

ClinVar, 26 in OMIM, and 81 in UniProt databases. 
respective enzyme activity are described in table S3. All studies presented the CYP21A2 activity measured by the hydroxylation of $170 \mathrm{HP}$, while 86 also measured progesterone hydroxylation.

We obtained 22 SNVs in the CYP21A2 gene with the correct prediction for all tested predictors, although the exact number was incorrectly predicted for at least half of them (Table S3). There was no SNV wrongly predicted by all predictors. We compared the hit and miss by the 17 predictors for all SNV affecting CYP21A2 activity, the CAH group, and for all the non-pathogenic SNV of the neutral group. We showed that $22 \%$ (22 of 90 ) SNVs from the CAH group obtained the correct score by all predictors, while $23 \%$ ( 21 of 90 ) were wrongly predicted by at least nine tools. The neutral group got 2 of its 13 SNVs (15.4\%) rightly mis predicted by all and one by nine predictors. Moreover, we divided the SNV of the CAH group into the CL and NC groups. We got $37 \%$ (out of $51 \mathrm{SNVs}$ ) from the $\mathrm{CL}$ and CL and NC groups, respectively, were wrongly predicted by nine tools. 
(NPVs) higher than 0.5. PANTHER-PSEP has no result for the NPV, as it could not identify benign variants.

We obtained both sensibility and specificity higher than 0.8 for three predictors, CADD (se=0.96 and $\mathrm{sp}=0.85$ ), ConSurf ( $\mathrm{se}=0.88$ and $\mathrm{sp}=0.90)$, and PolyPhen-2 ( $\mathrm{se}=0.87$ and $\mathrm{sp}=0.85)$. Moreover, five predictors obtained the accuracy between excellent and good, CADD (0.94), PANTHER-PSEP (0.91), DANN (0.89), ConSurf (0.88), and PolyPhen-2 (0.86) (Table).

The Matthews' correlation coefficient (MCC) test showed positive values for that of almost all predictors (except by PANTHER-PSEP), being five of them with the MCC $>0.5$ (Table). The best performance, value closer to +1 , was obtained by CADD ( 0.75$)$, followed by ConSurf ( 0.58$)$, PolyPhen2 (0.57), DANN (0.56), and PROVEN (0.51). Figure 3 shows a Venn diagram of the four predictors with better accuracy and MCC values.

Performance of predictors to identify SNVs affecting the specific CAH groups

We analyzed the performance of the selected predictors to identify 51 SNVs of the CL group and 39 SNVs of the NC group against 13 SNVs with a neutral effect (Table). Seventeen predictors obtained an excellent PPV rate $(>0.90)$ for the SNV CL group, 15 for the SNVs NC group. Four tools obtained excellent-good (> 0.8) NPV values for the CL group: CADD (1.0), DANN (0.9), PolyPhen-2 (0.85), and showed NPV >0.5.

Taking the sensibility and specificity balance, we obtained 12 tools with excellent-good values $(>0.8)$ for the $\mathrm{CL}$ group (Table). However, for the NC group, only CADD ( $\mathrm{se}=0.9$ and $\mathrm{sp}=0.85$ ) obtained both sensibility and specificity with excellent-good values (Table). The accuracy was excellent for 7 predictors on CL group, CADD (0.97), ConSurf (0.95), PolyPhen-2 and PROVEN (0.94), DANN and 

for the $\mathrm{CL}$ group and $\mathrm{MCC}=0.71$ for the $\mathrm{NC}$ group.

\section{Discussion}

Genetics analysis is an essential approach for elucidating complex CYP21A2 deficiency cases, mainly to confirm asymptomatic carriers and unfollow false-positive cases ${ }^{7,16}$. Therefore, fast and accessible tools to infer variants' pathogenicity are essential to quickly deduce the harm of unknown variants. In silico prediction is one of the most accessible tools to infer the pathogenicity of SNVs.

Here, for the first time, we analyzed the performance of in silico prediction tools to discriminate pathogenic and neutral variants of the CYP21A2. We focus on the performance of 13 single predictors and four meta predictors chosen accordingly with the popularity and performance of free access programs. All these programs were able to identify pathogenic variants. Nonetheless, only PANTHERPSEP could not distinguish neutral variants, which is unacceptable for testing variants of the CYP21A2. Moreover, all tools showed better performance with variants of the CL group than the NC group, as expected, since the $C L$ group gathers the most harmful variants.

Our databank for performance tests comprises all missense variants of the CYP21A2 that are functionally characterized. With this strategy, we could get a more realistic result on the prediction evaluation. However, the number of variants was imbalanced between the two categories, 90 pathogenic and 13 neutral. Therefore, the primary statistics data considered for the performance 
sensibility and specificity are calculated with half of the information, they cannot represent all the performance by themselves, so we considered the sensibility-specificity balance. Additionally, we also calculated PPV and NPV but, as both are more sensitive to data disbalancing, they were not considered for the program performance ${ }^{34}$.

The main feature assessed by most single predictors tested is the evolutionary data since residue conservation over time can indicate critical residues for the protein function. Four of the tools tested use only this feature for the prediction calculation, FATHMM ${ }^{20}$, PhD-SNPg $^{24}$, PROVEAN $^{26}$, and SIFT ${ }^{27}$. A similar performance was obtained between these four tools, with a fair accuracy ranging from 0.73 (FATHMM) to 0.79 (SIFT), and MCC from 0.42 (PhD-SNPg) to 0.51 (PROVEAN). Additionally, SIFT had the most excellent sensibility-specificity balance between programs, similar to the performance shown previously ${ }^{27}$. In another study ${ }^{14}$, with HSD17B3, NR5A1, AR, and LHCGR genes, SIFT and PROVEAN also had the same performance, with an accuracy of $0.74-0.75$, and MCC of 0.5 . In yet another study ${ }^{12}$, SIFT and PROVEN showed the best results between nine programs tested for GJB2, GJB6, and GJB3 genes, with an accuracy of 0.89 , while FATHMM produced a large number of erroneous predictions with an accuracy of 0.33 . FATHMM also had poor performance in another ${ }^{14}$ study, with an accuracy of 0.56 and MCC of 0.04 .

Changes in the secondary and tertiary structure by missense mutations are likely to affect the protein activity ${ }^{18}$. Therefore, it is no surprise that the second most evaluated feature is the structural information, being present in ConSurf ${ }^{18}$, MutPred2 ${ }^{22}$, PolyPhen2 ${ }^{10}$, and SNAP2 ${ }^{28}$. In addition, ConSurf includes phylogenetics relationships, MutPred2 functional proprieties, and SNAP2 uses a matrix of effect probabilities with a neural network method ${ }^{18,22,28}$. While, PolyPhen2 has two trained datasets as options, HumVar and HumDir. The first trained dataset is suggested for diagnostics of Mendelian diseases, which requires variants with a drastic difference effect ${ }^{25}$. Moreover, PolyPhen2 has a low 
than reported in ${ }^{10}$. ConSurf obtained similar performance with the setting used in or test $(a c=0.88$, MCC=0.58). Since the ConSurf setting is chosen for the alignment sequences, databank, and algorithms, the performance is not comparable with other studies, as it was reported by ConSurf's developers ${ }^{18}$. On the other hand, MutPred2 and SNAP2 both had sensibility-specificity imbalanced of 1.4 and 1.5 -folds, respectively, and almost the same fair performance. However, these values were relatively better than reported for MutPred 2 by ${ }^{22}$ with ClinVar and UniProt database and SNAP2 by ${ }^{28}$ with a databank with more than 9,500 variants from human genes.

For meta predictors, which work with many databases and combine outputs from other predictors to generate their own, we expected to obtain one of the best performances. However, counterintuitively, our study showed an intermediate performance compared with the single predictors tested, and the number of tools combined was not related to the prediction improvement. Meta-SNP and PredictSNP performance were better than PredictSNP2 and S3Ds\&GO. Furthermore, comparing with the developer tests, we obtained for Meta-SNP ${ }^{32}$ and PredictSNP ${ }^{30}$ a similar accuracy, while the performance for PredictSNP2 ${ }^{31}$ and S3Ds\&GO ${ }^{33}$ were lower. Meta-SNP and PredictSNP share three single predictors, PhD-SNP, SNAP, and SIFT.

Therefore, for the CYP21A2 variants tested, the best performance to categorize missense variants pathogenicity was CADD, with an overall accuracy of 0.94 (CL 0.97; NC 0.88) and MCC of 0.75 (CL 0.9, NC 0.71). The specificity (0.85) and sensibility (0.9) also got a good balance. Interestingly, the accuracy and specificity obtained for CYP21A2 were even higher than reported by the software developers ${ }^{17}$ with the ClinVar database, which was 0.85 and 0.57 , respectively. ConSurf, DANN, and PolyPhen-2 showed a similar performance, holding the second-best results accordingly with the accuracy (CAH group 0.86-0.89; CL 0.92-0.95; NC 0.77-0.81,) and MCC (CAH group 0.56-0.58; CL 0.75-0.83; NC 0.510.56) values. The sensibility and specificity for ConSurf and PolyPhen2 were well balanced, while DANN had 1.3 -folds less specificity than sensibility. The original article of DANN ${ }^{19}$ presents only the area under the curve (AUC) ROC, which was 0.95 using the ClinVar database for the performance test. 
PolyPhen 2 showed better sensibility-specificity balance for the CYP21A2 variants than the values presented by ${ }^{10}$, testing the tool with gene-specific mutations (BRCA1, MSH2, MLH1, and TP52). We obtained a sensibility similar to a previous analysis ${ }^{10}$, but the specificity was lower, 0.85 and 0.60 , respectively.

The individual error of the top four predictors was six on CADD, 12 on ConSurf, 11 on DANN, and 14 on PolyPhen2. However, computing their prediction together, we would have four false results from 103 missense SNV in the CPY21A2, one neutral (p.L13M), and three pathogenic from the NC phenotype group (p.P106L, p.R225W, and p.M474I). The variant p.P106L was correctly categorized by SNAP2 and PANTHER-PSEP. In turn, PROVEAN ${ }^{26}$ could type the other three variants correctly, even with lower sensibility value than the other four tools, mainly for the pathogenic SNVs of the NC group (0.49). Nonetheless, we obtained an intermediated performance with PROVEAN ( $a c=0.77 ; M C C=0.51$ ), which could be because it uses the neighborhood sequences as input, which can be a trick for enzymes since they have some residues with high conservation making critical connections between variable residues. Comparing with the developer' test ${ }^{26}$ with the UniProt database ( $\left.s e=0.78 ; s p=0.79\right)$, we had imbalanced sensibility-specificity imbalanced, with similar overall sensibility (0.73) and higher specificity (1.0).

In conclusion, we could identify CADD, ConSurf, DANN, and PolyPhen2 as good programs for missense variants prediction of the CYP21A2. Moreover, CADD had the best performance also for identifying mild mutations from the NC group, followed by ConSurf and DANN. These results may be applicable in future analysis of new or uncharacterized missense variants of the CYP21A2.

\section{Author Contributions:}

Conceptualization, M.J.P, R.L-B, A.Z., and A.V.P; methodology, M.J.P., A.V.P, and R.L-B; formal analysis, M.J.P; investigation, M.J.P.; resources, A.V.P. and A.Z.; writing-original draft preparation, 
341 M.J.P.; writing-review and editing, R.L-B., A.Z, L.M.R., and A.V.P.; visualization, M.J.P; supervision,

342 A.V.P., A.Z., M.L.R.R., and R.L-B.; project administration, xx; funding acquisition, M.J.P., A.V.P., and

343 A.Z. All authors have read and agreed to the published version of the manuscript.

345 Supplementary Materials:

346 Supplementary materials can be accessed at http://dx.doi.org/10.48350/162936 and

347 https://boris.unibe.ch/id/eprint/162936 
1. Miller WL, Auchus RJ. The molecular biology, biochemistry, and physiology of human steroidogenesis and its disorders. Endocrine Reviews. 2011;32(1):81-151. doi:10.1210/er.2010-0013

2. Witchel SF. Congenital Adrenal Hyperplasia. Journal of Pediatric and Adolescent Gynecology. 2017;30(5):520-534. doi:10.1016/j.jpag.2017.04.001

3. New MI, Abraham M, Gonzalez B, et al. Genotype-phenotype correlation in 1,507 families with congenital adrenal hyperplasia owing to 21-hydroxylase deficiency. Proceedings of the National Academy of Sciences of the United States of America. 2013;110(7):2611-2616. doi:10.1073/pnas.1300057110

4. Simonetti L, Bruque CD, Fernández CS, et al. CYP21A2 mutation update: Comprehensive analysis of databases and published genetic variants. Human Mutation. 2018;39(1):5-22. doi:10.1002/humu.23351

5. Rodrigues NR, Dunham I, Yu Y, Carroll' MC, Porter2 RR, Campbell RD. Molecular Characterization of the HLA-Linked Steroid 21-Hydroxylase B Gene from an Individual with

7. Baumgartner-Parzer S, Witsch-Baumgartner $\mathrm{M}$, Hoeppner W. EMQN best practice guidelines

8. Lee C yu, Yen HY, Zhong AW, Gao H. Resolving misalignment interference for NGS-based

9. Gangodkar $\mathrm{P}$, Khadilkar V, Raghupathy $\mathrm{P}$, et al. Clinical application of a novel next generation sequencing assay for CYP21A2 gene in 310 cases of 21- hydroxylase congenital adrenal hyperplasia from India. Endocrine. 2021;71(1):189-198. doi:10.1007/s12020-020-02494-z

10. Hicks S, Wheeler DA, Plon SE, Kimmel M. Prediction of missense mutation functionality depends on both the algorithm and sequence alignment employed. Human Mutation. 2011;32(6):661-668. doi:10.1002/humu.21490

11. Tang B, Li B, Gao LD, et al. Optimization of in silico tools for predicting genetic variants: individualizing for genes with molecular sub-regional stratification. Briefings in Bioinformatics. 2020;21(5):1776-1786. doi:10.1093/bib/bbz115

12. Pshennikova VG, Barashkov NA, Romanov GP, et al. Comparison of Predictive in Silico Tools on Missense Variants in GJB2, GJB6, and GJB3 Genes Associated with Autosomal Recessive Deafness 1A (DFNB1A). Scientific World Journal. 2019;2019. doi:10.1155/2019/5198931

13. Hart SN, Polley EC, Shimelis H, Yadav S, Couch FJ. Prediction of the functional impact of missense variants in BRCA1 and BRCA2 with BRCA-ML. npj Breast Cancer. 2020;6(1):13. doi:10.1038/s41523-020-0159-x 
14. Montenegro LR, Lerário AM, Nishi MY, Jorge AAL, Mendonca BB. Performance of mutation pathogenicity prediction tools on missense variants associated with 46 ,xy differences of sex development. Clinics. 2021;76:1-5. doi:10.6061/clinics/2021/e2052

15. Khan $\mathrm{S}$, Vihinen $\mathrm{M}$. Spectrum of disease-causing mutations in protein secondary structures. BMC Structural Biology. 2007;7. doi:10.1186/1472-6807-7-56

16. Pignatelli D, Carvalho BL, Palmeiro A, Barros A, Guerreiro SG, Maçut D. The complexities in genotyping of congenital adrenal hyperplasia: 21-hydroxylase deficiency. Frontiers in Endocrinology. 2019;10(JULY). doi:10.3389/fendo.2019.00432

17. van der Velde KJ, de Boer EN, van Diemen CC, et al. GAVIN: Gene-Aware Variant INterpretation for medical sequencing. Genome Biology. 2017;18(1). doi:10.1186/s13059016-1141-7

18. Ashkenazy H, Abadi S, Martz E, et al. ConSurf 2016: an improved methodology to estimate and visualize evolutionary conservation in macromolecules. Nucleic Acids Research. 2016;44(W1):W344-W350. doi:10.1093/nar/gkw408

19. Quang D, Chen Y, Xie X. DANN: A deep learning approach for annotating the pathogenicity of genetic variants. Bioinformatics. 2015;31(5):761-763. doi:10.1093/bioinformatics/btu703

20. Shihab HA, Gough J, Cooper DN, et al. Predicting the Functional, Molecular, and Phenotypic Consequences of Amino Acid Substitutions using Hidden Markov Models. Human Mutation. 2013;34(1):57-65. doi:10.1002/humu.22225

21. Stone EA, Sidow A. Physicochemical constraint violation by missense substitutions mediates impairment of protein function and disease severity. Genome Research. 2005;15(7):978-986. doi:10.1101/gr.3804205

22. Pejaver $\mathrm{V}$, Urresti J, Lugo-Martinez J, et al. Inferring the molecular and phenotypic impact of amino acid variants with MutPred2. Nature Communications. 2020;11(1). doi:10.1038/s41467-020-19669-x

23. Tang $\mathrm{H}$, Thomas PD. PANTHER-PSEP: Predicting disease-causing genetic variants using position-specific evolutionary preservation. Bioinformatics. 2016;32(14):2230-2232. doi:10.1093/bioinformatics/btw222

24. Capriotti E, Fariselli P. PhD-SNPg: A webserver and lightweight tool for scoring single nucleotide variants. Nucleic Acids Research. 2017;45(W1):W247-W252. doi:10.1093/nar/gkx369

25. Adzhubei I, Jordan DM, Sunyaev SR. Predicting Functional Effect of Human Missense Mutations Using PolyPhen-2. Current Protocols in Human Genetics. 2013;76(1). doi:10.1002/0471142905.hg0720s76

26. Choi Y, Sims GE, Murphy S, Miller JR, Chan AP. Predicting the Functional Effect of Amino Acid Substitutions and Indels. PLOS ONE. 2012;7(10). doi:10.1371/journal.pone.0046688

27. Vaser R, Adusumalli S, Leng SN, Sikic M, Ng PC. SIFT missense predictions for genomes. Nature Protocols. 2016;11(1):1-9. doi:10.1038/nprot.2015.123

28. Hecht $\mathrm{M}$, Bromberg $\mathrm{Y}$, Rost $\mathrm{B}$. Better prediction of functional effects for sequence variants. BMC Genomics. 2015;16(S8):S1. doi:10.1186/1471-2164-16-S8-S1 
29. Calabrese R, Capriotti E, Fariselli P, Martelli PL, Casadio R. Functional annotations improve the predictive score of human disease-related mutations in proteins. Human Mutation. 2009;30(8):1237-1244. doi:10.1002/humu.21047

30. Bendl J, Stourac J, Salanda O, et al. PredictSNP: Robust and Accurate Consensus Classifier for Prediction of Disease-Related Mutations. PLoS Computational Biology. 2014;10(1). doi:10.1371/journal.pcbi.1003440

31. Bendl J, Musil M, Štourač J, Zendulka J, Damborský J, Brezovský J. PredictSNP2: A Unified Platform for Accurately Evaluating SNP Effects by Exploiting the Different Characteristics of Variants in Distinct Genomic Regions. PLoS Computational Biology. 2016;12(5). doi:10.1371/journal.pcbi.1004962

32. Capriotti E, Altman RB, Bromberg Y. Collective judgment predicts disease-associated single nucleotide variants. BMC genomics. 2013;14 Suppl 3. doi:10.1186/1471-2164-14-s3-s2

33. Capriotti E, Altman RB. Improving the prediction of disease-related variants using protein three-dimensional structure. BMC Bioinformatics. 2011;12(SUPPL. 4). doi:10.1186/14712105-12-S4-S3

34. Vihinen M. How to evaluate performance of prediction methods? Measures and their interpretation in variation effect analysis. BMC genomics. 2012;13 Suppl 4. doi:10.1186/14712164-13-S4-S2

35. Chicco D, Tötsch N, Jurman G. The matthews correlation coefficient (Mcc) is more reliable than balanced accuracy, bookmaker informedness, and markedness in two-class confusion matrix evaluation. BioData Mining. 2021;14:1-22. doi:10.1186/s13040-021-00244-z 


\section{TABLES LEGENDS}

453 Table 1. Performance of 17 programs to predict the effect SNVs in the CYP21A2. We performed the

454 analysis with 103 functionally characterized variants, 90 damaging the protein functionality, and 13

455 neutral-Color scores from blue (good result) to yellow (not good). a meta-predictor. nr, no result;

456 PPV, positive predictive value; NPV, negative predictive value; Se, sensibility; Sp, specificity; Ac,

457 accuracy; MCC, Matthews' correlation coefficient test.

Table 2. Performance of 17 programs for the specific CYP21A2 groups. We predict the effect of SNVs in the CYP21A2 dividing them by the two levels of protein damage: the severe (classical mutation, $\mathrm{CL}$ group) and mild (non-classical mutation, NC group). We performed the analysis with 103 SNVs of known effect, 51 being CL, $39 \mathrm{NC}$, and 13 neutral. Color score from blue (good result) to yellow (not good). ${ }^{a}$ meta-predictor. nr, no result; PPV, positive predictive value; NPV, negative predictive value; Se, sensibility; Sp, specificity; Ac, accuracy; MCC, Matthews' correlation coefficient test. 


\section{FIGURE LEGENDS}

466 Figure 1. Composition of the four meta-predictors studied. The PredictSNP algorithm comprises

467 outputs of six single-predictors, the PredictSNP2 of six, the Meta-SNP of 4, and the S3Ds\&GO of three 468 predictors.

469

470 Figure 2. Frequency of hit and miss obtained for each SNV by mutation group. Each SNV (vertical list)

471 was analyzed by seventeen predictors (horizontal measurement) performed with the default setting

472 for missense mutation. Please, refer to Table S4-S6 for details. TP, true positive; TN, true negative; FN,

473 false negative; FP, false positive.

474

475 Figure 3. Venn diagram indicating overlaps of the hit for four predictors with good-excellent 476 performance for SNVs on CYP21A2. Total, indicate all 103 SNVs tested. (Image generated with 477 https://bioinformatics.psb.ugent.be/webtools/Venn/). 
478 Table 1. Performance of 17 programs to predict the effect SNVs in the CYP21A2. We performed the

479 analysis with 103 functionally characterized variants, 90 damaging the protein functionality, and 13

480 neutral-Color scores from blue (good result) to yellow (not good).

\begin{tabular}{|c|c|c|c|c|c|c|c|c|c|c|}
\hline Predictors & TP & FN & TN & FP & PPV & NPV & Se & Sp & $A c$ & $\mathrm{MCC}$ \\
\hline PredictSNP ${ }^{a}$ & 58 & 32 & 13 & 0 & 1.00 & 0.29 & 0.64 & 1.00 & 0.69 & 0.43 \\
\hline PredictSNP2 $^{\mathrm{a}}$ & 44 & 46 & 13 & 0 & 1.00 & 0.22 & 0.49 & 1.00 & 0.55 & 0.33 \\
\hline Meta-SNPa & 60 & 30 & 13 & 0 & 1.00 & 0.30 & 0.67 & 1.00 & 0.71 & 0.45 \\
\hline S3Ds\&GOa & 58 & 32 & 8 & 0 & 1.00 & 0.20 & 0.64 & 1.00 & 0.67 & 0.36 \\
\hline CADD & 86 & 4 & 11 & 2 & 0.98 & 0.73 & 0.96 & 0.85 & 0.94 & 0.75 \\
\hline ConSurf & 79 & 11 & 9 & 1 & 0.99 & 0.45 & 0.88 & 0.90 & 0.88 & 0.58 \\
\hline DANN & 83 & 7 & 9 & 4 & 0.95 & 0.56 & 0.92 & 0.69 & 0.89 & 0.56 \\
\hline FATHMM & 62 & 28 & 13 & 0 & 1.00 & 0.32 & 0.69 & 1.00 & 0.73 & 0.47 \\
\hline MAPP & 54 & 36 & 10 & 2 & 0.96 & 0.22 & 0.60 & 0.83 & 0.63 & 0.28 \\
\hline MutPred2 & 62 & 28 & 13 & 0 & 1.00 & 0.32 & 0.69 & 1.00 & 0.73 & 0.47 \\
\hline PANTHER-PSEP & 90 & 0 & 0 & 9 & 0.91 & $\mathrm{nr}$ & 1.00 & 0.00 & 0.91 & $\mathrm{nr}$ \\
\hline PhD-SNPg & 62 & 28 & 12 & 1 & 0.98 & 0.30 & 0.69 & 0.92 & 0.72 & 0.42 \\
\hline PolyPhen2-HumVar & 79 & 11 & 12 & 1 & 0.99 & 0.52 & 0.88 & 0.92 & 0.88 & 0.64 \\
\hline PROVEAN & 66 & 24 & 13 & 0 & 1.00 & 0.35 & 0.73 & 1.00 & 0.77 & 0.51 \\
\hline SIFT & 70 & 20 & 11 & 2 & 0.97 & 0.35 & 0.78 & 0.85 & 0.79 & 0.45 \\
\hline SNP2 & 59 & 31 & 13 & 0 & 1.00 & 0.30 & 0.66 & 1.00 & 0.70 & 0.44 \\
\hline SNPs\&GO & 54 & 36 & 13 & 0 & 1.00 & 0.27 & 0.60 & 1.00 & 0.65 & 0.40 \\
\hline
\end{tabular}

481

${ }^{a}$ meta-predictor. nr, no result; PPV, positive predictive value; NPV, negative predictive value; Se, 
Table 2. Performance of 17 programs for the specific CYP21A2 groups. We predict the effect of SNVs in the CYP21A2 dividing them by the two levels of protein damage: the severe (classical mutation, $\mathrm{CL}$ group) and mild (non-classical mutation, NC group). We performed the analysis with 103 SNVs of known effect, 51 being $\mathrm{CL}, 39 \mathrm{NC}$, and 13 neutral. Color score from blue (good result) to yellow (not good).

\begin{tabular}{|c|c|c|c|c|c|c|c|c|c|c|c|c|}
\hline \multirow{2}{*}{ Specifics groups } & \multicolumn{2}{|c|}{ PPV } & \multicolumn{2}{|c|}{ NPV } & \multicolumn{2}{|c|}{ Se } & \multicolumn{2}{|c|}{$S p$} & \multicolumn{2}{|c|}{ Ac } & \multicolumn{2}{|c|}{ MCC } \\
\hline & $\mathrm{Cl}$ & NC & $\mathrm{Cl}$ & NC & $\mathrm{Cl}$ & NC & $\mathrm{Cl}$ & NC & $\mathrm{Cl}$ & NC & $\mathrm{Cl}$ & NC \\
\hline PredictSNP ${ }^{a}$ & 1.00 & 1.00 & 0.65 & 0.34 & 0.86 & 0.36 & 1.00 & 1.00 & 0.89 & 0.52 & 0.75 & 0.35 \\
\hline PredictSNP2 ${ }^{a}$ & 1.00 & 1.00 & 0.39 & 0.33 & 0.61 & 0.33 & 1.00 & 1.00 & 0.69 & 0.50 & 0.49 & 0.33 \\
\hline Meta-SNPa & 1.00 & 1.00 & 0.68 & 0.35 & 0.88 & 0.38 & 1.00 & 1.00 & 0.91 & 0.54 & 0.78 & 0.37 \\
\hline S3Ds\&GOa & 1.00 & 1.00 & 0.53 & 0.24 & 0.86 & 0.36 & 1.00 & 1.00 & 0.88 & 0.47 & 0.68 & 0.29 \\
\hline CADD & 0.96 & 0.95 & 1.00 & 0.73 & 1.00 & 0.90 & 0.85 & 0.85 & 0.97 & 0.88 & 0.90 & 0.71 \\
\hline ConSurf & 0.98 & 0.97 & 0.82 & 0.50 & 0.96 & 0.77 & 0.90 & 0.90 & 0.95 & 0.80 & 0.83 & 0.56 \\
\hline DANN & 0.93 & 0.89 & 0.90 & 0.60 & 0.98 & 0.85 & 0.69 & 0.69 & 0.92 & 0.81 & 0.75 & 0.51 \\
\hline FATHMM & 1.00 & 1.00 & 0.52 & 0.45 & 0.76 & 0.59 & 1.00 & 1.00 & 0.81 & 0.69 & 0.63 & 0.51 \\
\hline MAPP & 0.95 & 0.88 & 0.48 & 0.29 & 0.78 & 0.36 & 0.83 & 0.83 & 0.79 & 0.47 & 0.51 & 0.18 \\
\hline MutPred2 & 1.00 & 1.00 & 0.72 & 0.36 & 0.90 & 0.41 & 1.00 & 1.00 & 0.92 & 0.56 & 0.81 & 0.38 \\
\hline PANTHER-PSEP & 0.85 & 0.81 & $\mathrm{nr}$ & $\mathrm{nr}$ & 1.00 & 1.00 & 0.00 & 0.00 & 0.85 & 0.81 & $\mathrm{nr}$ & $\mathrm{nr}$ \\
\hline PhD-SNPg & 0.98 & 0.95 & 0.57 & 0.39 & 0.82 & 0.51 & 0.92 & 0.92 & 0.84 & 0.62 & 0.64 & 0.38 \\
\hline PolyPhen2-HumVar & 0.96 & 0.94 & 0.85 & 0.52 & 0.96 & 0.74 & 0.85 & 0.85 & 0.94 & 0.77 & 0.81 & 0.52 \\
\hline PROVEAN & 1.00 & 1.00 & 0.76 & 0.39 & 0.92 & 0.49 & 1.00 & 1.00 & 0.94 & 0.62 & 0.84 & 0.44 \\
\hline SIFT & 0.96 & 0.92 & 0.69 & 0.42 & 0.90 & 0.62 & 0.85 & 0.85 & 0.89 & 0.67 & 0.70 & 0.40 \\
\hline SNP2 & 1.00 & 1.00 & 0.59 & 0.37 & 0.82 & 0.44 & 1.00 & 1.00 & 0.86 & 0.58 & 0.70 & 0.40 \\
\hline SNPs\&GO & 1.00 & 1.00 & 0.59 & 0.33 & 0.82 & 0.31 & 1.00 & 1.00 & 0.86 & 0.48 & 0.70 & 0.32 \\
\hline
\end{tabular}




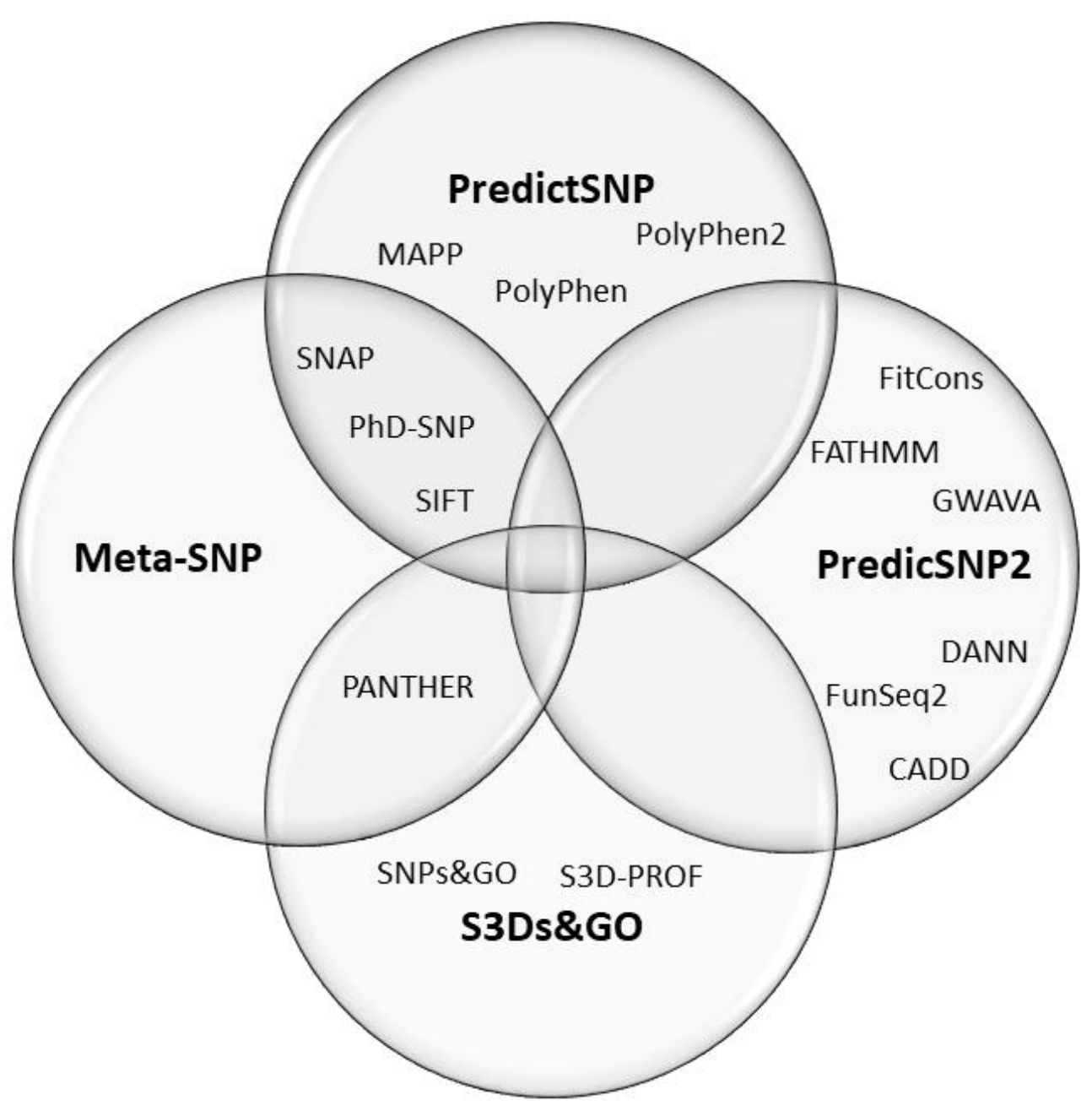


bioRxiv preprint doi: https://doi.org/10.1101/2021.12.21.473700; this version posted December 23, 2021. The copyright holder for this preprint (which was not certified by peer review) is the author/funder. All rights reserved. No reuse allowed without permission.

\section{TP Group: Classical}

$\begin{array}{lllllllllll}0 & 1 & 2 & 3 & 4 & 5 & 6 & 7 & 8 & 9 & 1011 \\ 12 & 1314151617\end{array}$

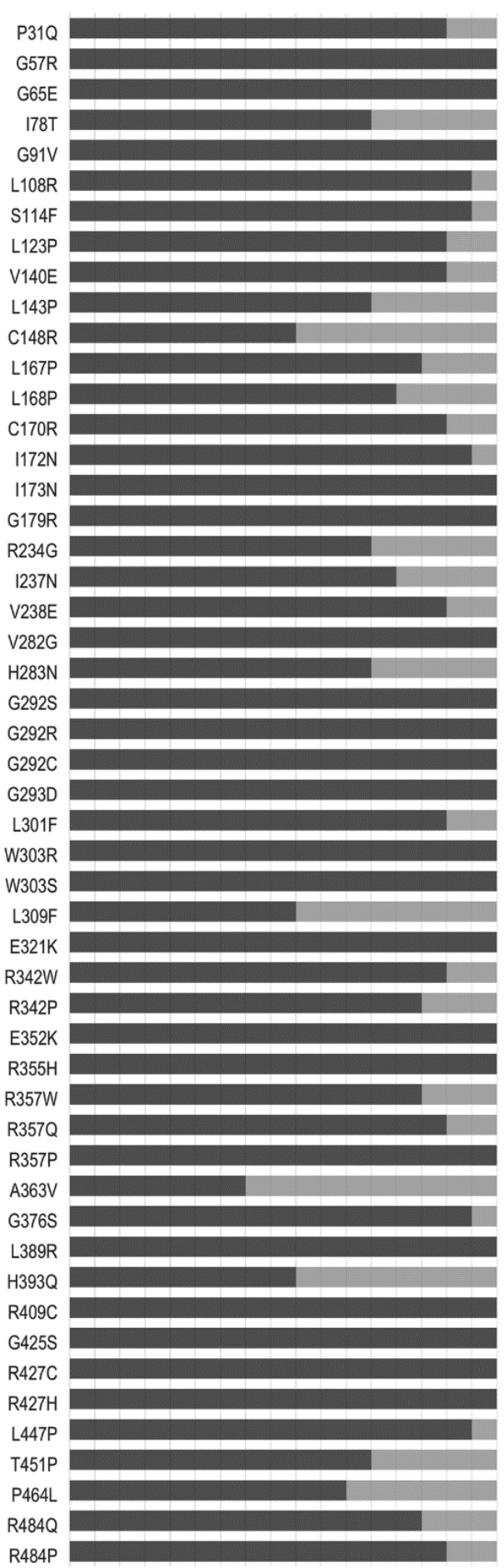

FN TP Group: Nonclassical FN

01233456678891011121314151617

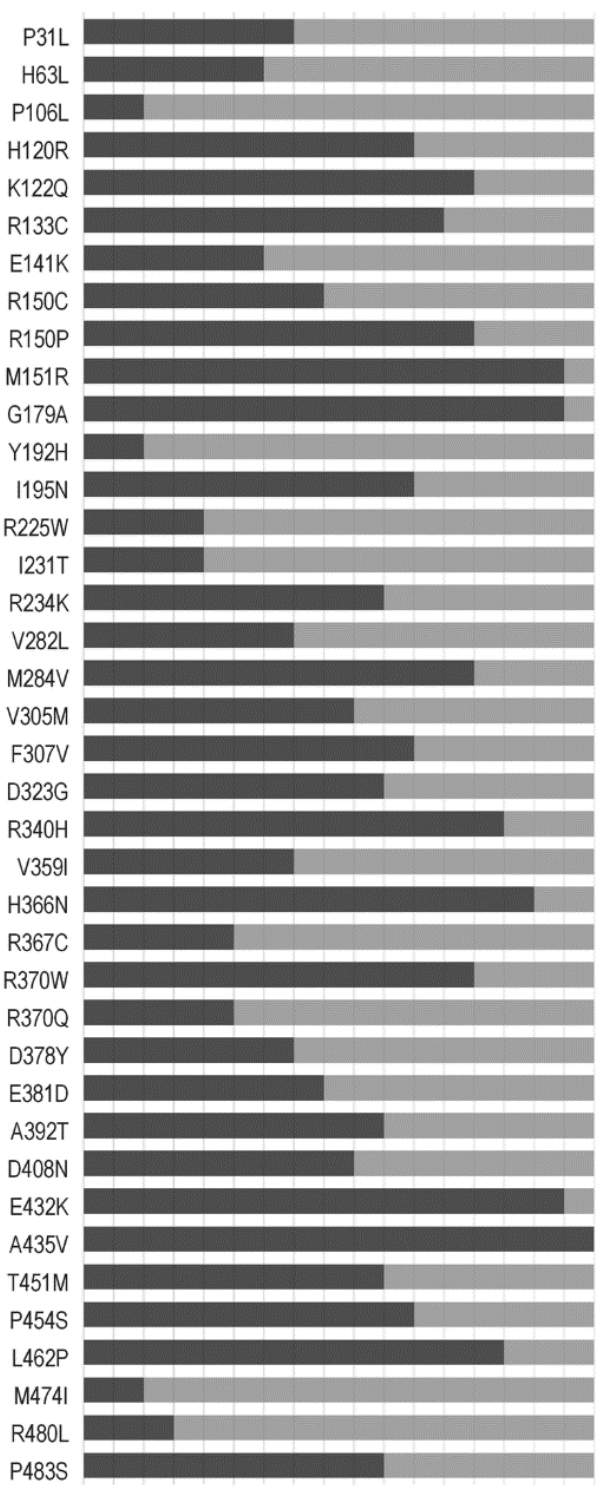

TN Group: Neutral FP $0 \begin{array}{llllllllll}2 & 2 & 4 & 5 & 6 & 7 & 8 & 9 & 1011121314151617\end{array}$

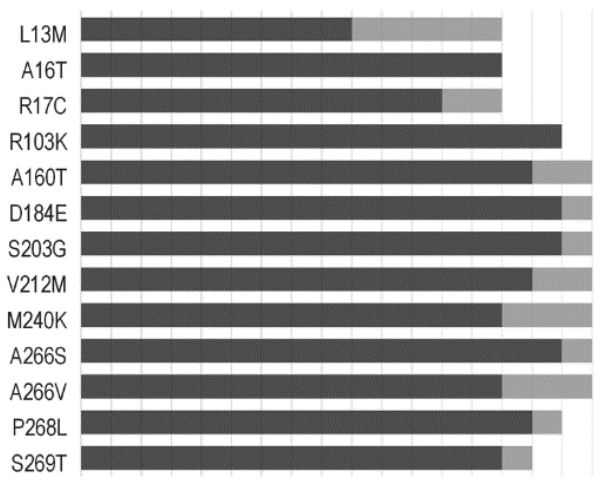




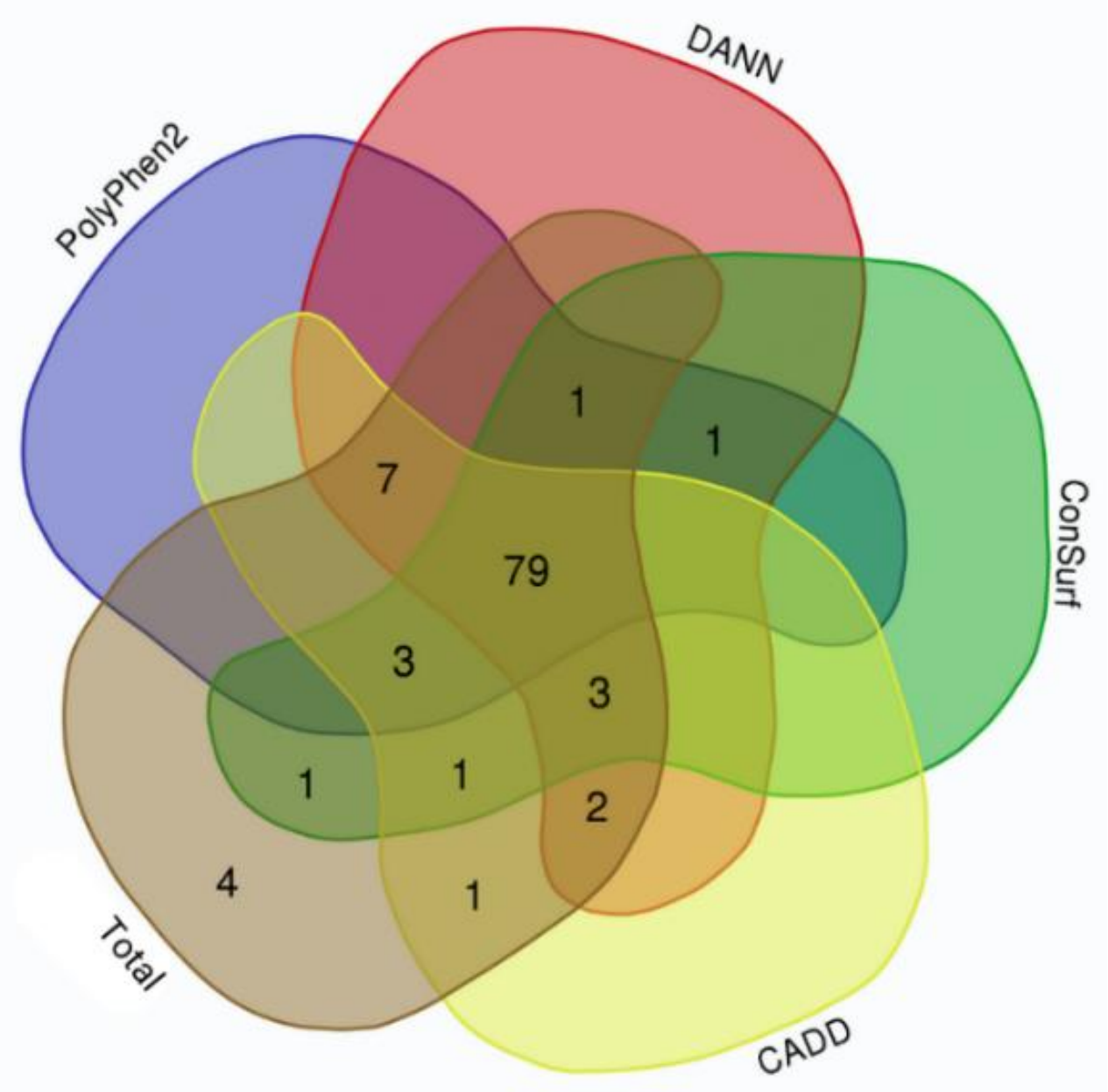

